\title{
Comparison of pre-operative dGEMRIC imaging with intra-operative findings in femoroacetabular impingement: preliminary findings
}

\author{
Bernd Bittersohl • Harish S. Hosalkar • \\ Sebastian Apprich • Stefan A. Werlen • \\ Klaus A. Siebenrock • Tallal Charles Mamisch
}

Received: 13 May 2010 /Revised: 8 August 2010 /Accepted: 19 September 2010 /Published online: 13 October 2010

(C) ISS 2010

\begin{abstract}
Objectives To study standard MRI and dGEMRIC in patients with symptomatic FAI undergoing surgical intervention and compare them with intra-operative findings to see if they were corroborative.

Methods Sixteen patients with symptomatic FAI that warranted surgical intervention were prospectively studied. All patients underwent plain radiographic series for FAI assessment followed by standard MRI and dGEMRIC. Subsequently, patients were surgically treated with safe dislocation and the joint was evaluated for any macroscopic signs of damaged cartilage. Data were statistically analyzed. Results A total of 224 zones in 16 patients were evaluated. One hundred and sixteen zones were intra-operatively rated as normal with mean $\mathrm{T} 1$ values of $510.1 \mathrm{~ms} \pm 141.2 \mathrm{~ms}$. Eighty zones had evidence of damage with mean T1 values of $453.1 \mathrm{~ms} \pm 113.6 \mathrm{~ms}$. The difference in these $\mathrm{T} 1$ values was significant $(p=0.003)$. Correlation between standard MRI and intra-operative findings was moderate $(r=0.535$, $p<0.001)$. Intra-operative findings revealed more damage than standard MRI. On standard MRI, $68.6 \%$ zones were graded normal while $31.4 \%$ had evidence of damage. On
\end{abstract}

B. Bittersohl · S. Apprich · K. A. Siebenrock · T. C. Mamisch ( $ه)$ Department of Orthopedic Surgery, University of Bern,

Inselspital,

3010 Bern, Switzerland

e-mail: mamisch@bwh.harvard.edu

H. S. Hosalkar

Department of Orthopedic Surgery, Children's Hospital,

San Diego, CA, USA

S. A. Werlen

Department of Radiology, Sonnenhof Hospital,

Bern, Switzerland intra-operative visualization, $56.4 \%$ zones were graded normal and $43.6 \%$ had evidence of damage. Correlation between dGEMRIC and intra-operative findings turned out to be weak $(r=0.114, p<0.126)$. On T1 assessment $31.4 \%$ of zones were graded as normal and $68.6 \%$ as damaged. Conclusions dGEMRIC was significantly different between normal and affected cartilage based on intra-operative assessment. The correlation for morphological findings was limited, underestimating defects. By combining morphological with biochemical assessment dGEMRIC may play some role in the future to prognosticate outcomes and facilitate surgical planning and intervention.

Keywords MRI $\cdot$ Hip $\cdot$ Cartilage $\cdot$ FAI $\cdot$ dGEMRIC $\cdot$ Surgery

\section{Introduction}

Femoroacetabular impingement (FAI) results from an impaction between the proximal femur and the acetabular rim that is induced by an abnormal morphology of the femoral head-neck region (cam-FAI), the acetabulum (pincer-FAI) or both (mixed-FAI). Mixed-FAI type is the most common form. Cam-FAI results from a non-spherical shape of the femoral head that may be combined with poor head-neck offset. While entering the hip joint during flexion and internal rotation, the femoral head-neck junction abuts anterior-superior in the acetabular rim causing labral damage, cartilage shearing, and separation among cartilage, the labrum, and the subchondral bone. Pincer-FAI occurs due to acetabular over-coverage or improper configuration and shape of the acetabulum.

Untreated symptomatic FAI is a risk factor for the development of premature osteoarthritis (OA) in the hip 
[1-3]. Multiple reports have shown good outcomes following early surgical intervention in patients with preexisting mild-changes of $\mathrm{OA}$, but poor results in patients with advanced degenerative changes $[2,4,5]$. Therefore, it is understandably critical for the treating clinician to be able to detect cartilage changes of damage and degeneration not only in detail, but also at an early stage to maximize patient benefit.

Currently, routine magnetic resonance imaging (MRI) and/or MR arthrography, which use intra-articular injected contrast medium gadolinium for better depiction of labral and cartilage defects, remain the imaging techniques of choice [6]. MR arthrography has been proved to be superior to routine MRI, for accurate and reliable diagnosis of labral pathologies [7-10]. Despite these advantages the ability of MR arthrography to detect varying grades of cartilage damage is fairly limited. Also, based on the technology of these current techniques, early histological and biochemical changes at the beginning of OA cannot be detected.

The MRI technique of delayed gadolinium-enhanced MRI of cartilage (dGEMRIC) is a histologically validated $[11,12]$ and well-documented [13-24] method that is sensitive to the charge density of cartilage contributed by glycosaminoglycans (GAGs) [25, 26]. GAGs are important structural components of cartilage and relevant to maintaining the intrinsic mechanical properties that are lost early in the process of OA $[27,28]$. The dGEMRIC technique utilizes the anionic, negatively charged contrast agent gadolinium-diethylene triamine penta-acetic acid (GdDTPA $^{2-}$ ), which is also used as standard contrast agent for MR arthrography in the daily clinical set-up. Gd-DTPA ${ }^{2-}$ distributes within cartilage inversely to the negatively charged GAG content. Thus, the Gd-DTPA ${ }^{2-}$ concentration will be low in normal cartilage in contrast to the relatively high concentration of Gd-DTPA ${ }^{2-}$, which accumulates in degenerated cartilage due to the decreased GAG. GdDTPA $^{2-}$ shortens the T1 relaxation time. Thereby, subsequent $\mathrm{T} 1$ measurement described as the dGEMRIC index or T1Gd reflects the GAG content within a certain cartilage region [14]. Recently, fast $\mathrm{T} 1$ assessment utilizing dual flip angle (FA) gradient echo (GRE) in place of standard inversion recovery (IR) for T1 mapping has been validated in phantom studies and was used in vivo, enabling faster imaging times and three-dimensional (3D) T1 mapping [22, 29-31].

Thus, the dGEMRIC technique has the potential to detect early cartilage damage, which in turn is very helpful to clinicians for decision-making regarding timely intervention. To the best of our knowledge there are no reported studies in the literature that have compared the preoperative imaging findings of MRI using dGEMRIC for symptomatic FAI patients, with the subsequent intra- operative findings in the same cohort following safe surgical dislocation. The purpose of this present study was to prospectively study the standard MRI (morphological imaging) and dGEMRIC (biochemical imaging) findings in patients with symptomatic FAI undergoing surgical intervention and compare them with intra-operative findings to see if they were corroborative.

\section{Materials and methods}

Study population

Fifty consecutive patients with a diagnosis of FAI by history, clinical examination and X-ray underwent contrast MRI with additional dGEMRIC for this prospective study. The dGEMRIC index was compared in a preliminary study with the X-ray and MRI measurements [31]. Sixteen of these patients had surgery within 3 months and were included in this study. The mean time gap between imaging and surgery was 60.3 days \pm 13.9 days (range 47 days to 90 days).

There were 8 female and 8 male patients. Mean age \pm SD was $31 \pm 11.3$ years (range 17 to 57 years). Twelve hips involved the right side and 4 involved the left side. The degree of arthritis in 11 cases $(68.8 \%)$ was Tonnis grade 0 and in 4 cases Tonnis grade $1(25.0 \%)$. The mean Merle D'Aubigné scores were $15.4 \pm 1.4$ with a range of 13-17. Exclusion criteria such as any other hip pathology, previous hip surgery, advanced OA (Tonnis grade above 1), risks or contraindications for MRI including risks for Gd-DTPA ${ }^{2-}$ administration such as compromised renal function and allergies to contrast agent were ruled out. Potential side effects were clarified to the patients and their written informed consent obtained before the study was initiated. Patients in whom there was a time gap between the MRI and surgical intervention of more than 3 months were excluded. Thus, any possible dynamic and progressive effects of cartilage damage were minimized. The study was approved by the local ethics committee and written informed consent was given by each participant.

\section{MRI and dGEMRIC}

Magnetic resonance imaging was performed using a 1.5-T system (Magnetom Avanto; Siemens, Erlangen, Germany) and a body matrix-phased array coil, following 45 min after contrast administration $\left(0.4 \mathrm{ml} / \mathrm{kg}\right.$ Gd-DTPA ${ }^{2-}$, Magnevist ${ }^{\mathbb{}}$; BayerSchering AG, Germany) and 15 min of exercise before the start of the standard MR protocol to reach appropriate gadolinium infiltration [29, 31]. The coil was placed to examine each hip, ensuring that the device was accurately centered on top of the joint. The MRI protocol included the 
standard hip protocol and additionally a sequence for 3D T1 mapping:

1. Axial 2D turbo spin echo (TSE) with T1-weighting (repetition time (TR)/echo time (TE) $=491 \mathrm{~ms} / 13 \mathrm{~ms}, 3$ $\mathrm{mm}$ slice thickness, $160-\mathrm{mm}$ field of view (FOV), $512 \times 256$ matrix, acquisition time $(\mathrm{TA})=4.14 \mathrm{~min})$

2. Coronal oblique $2 \mathrm{D}$ TSE with proton-density (PD)weighting $(\mathrm{TR} / \mathrm{TE}=3,060 \mathrm{~ms} / 9.1 \mathrm{~ms}, 2-\mathrm{mm}$ slice thickness, $130-\mathrm{mm}$ FOV, $256 \times 205$ matrix, TA= $5.35 \mathrm{~min})$

3. Sagittal 2D TSE with PD-weighting $(\mathrm{TR} / \mathrm{TE}=$ 2,900 $\mathrm{ms} / 9.1 \mathrm{~ms}, 2-\mathrm{mm}$ slice thickness, $130-\mathrm{mm}$ FOV, $256 \times 205$ matrix, $\mathrm{TA}=5.35 \mathrm{~min}$ ),

4. Axial 2D fast low angle shot (FLASH) with T1weighting $(\mathrm{TR} / \mathrm{TE}=250 \mathrm{~ms} / 12 \mathrm{~ms}, 2-\mathrm{mm}$ slice thickness, $120-\mathrm{mm}$ FOV, $256 \times 205$ matrix, TA=3.52 min)

5. Radial 2D TSE with PD-weighting around the femoral neck and perpendicular to the acetabular rim $(\mathrm{TR} / \mathrm{TE}=1,800 \mathrm{~ms} / 13 \mathrm{~ms}, 4-\mathrm{mm}$ slice thickness, $180-\mathrm{mm}$ FOV, $512 \times 256$ matrix, $\mathrm{TA}=4.30 \mathrm{~min}$ ), and for T1-mapping

6. Dual FA 3D GRE volumetric interpolated breath-hold examination (VIBE) utilizing inline T1 measurement $\left(\mathrm{TR} / \mathrm{TE} / \mathrm{FA}=25 \mathrm{~ms} / 3.6 \mathrm{~ms} / 10^{\circ}\right.$ and $35^{\circ}, 0.78-\mathrm{mm}$ slice thickness, $200-\mathrm{mm}$ FOV, $256 \times 256$ matrix, voxel size $=$ $0.78 \mathrm{~mm}^{3}$ (isotropic), $\mathrm{slab}=96, \mathrm{TA}=8.46 \mathrm{~min}$ )

The complete examination, including contrast agent administration, standard MRI, and dual FA 3D GRE for T1 mapping, lasted approximately $90 \mathrm{~min}$.

\section{Standard MRI analysis}

Radial 2D TSE PD-weighted images around the femoral head were utilized to evaluate the presence of cartilage damage and were: anterior, anterior-superior, superioranterior, superior, superior-posterior, posterior-superior, and posterior (the former $=$ main direction). Within each radial image, cartilage was assessed in two zones: at the acetabular rim and centrally, close to the fovea, which included femoral and acetabular cartilage as one entity. Based on the observations, cartilage was graded as normal or damaged (surface irregularity, signal alteration, and tissue aberration) for each zone (central and peripheral).

\section{T1Gd analysis}

Analysis was performed by an experienced musculoskeletal radiologist who was blinded to patient findings and intraoperative results. For $\mathrm{T} 1$ analysis $\left(\mathrm{T} 1_{\mathrm{Gd}}\right.$, dGEMRIC index), the 3D data sets (morphological dual FA VIBE slab and resulting T1 maps) were transferred to a Leonardo ${ }^{\circledR}$ workstation (Siemens, Erlangen, Germany). Seven radial reformats concentric within the femoral head center, $30^{\circ}$ apart and perpendicular to the femoral neck were created by using multi-planar reconstruction (MPR) software. Thus, radial T1 maps were: anterior, anterior-superior, superioranterior, superior, superior-posterior, posterior-superior, and posterior (the former = main direction) resulted in further topographic $\mathrm{T} 1_{\mathrm{Gd}}$ measurement and comparison with standard MRI and intra-operative findings. Within each radial $\mathrm{T} 1$ map, $\mathrm{T} 1_{\mathrm{Gd}}$ was assessed in two zones: at the acetabular rim and centrally, close to the fovea, which included femoral and acetabular cartilage as one entity. We exercised care in drawing the region of interest (ROI) field within cartilage boundaries while not extending into bone tissue. Therefore, the corresponding morphological VIBE slab was equally processed in order to create seven identical radial reformats that served as anatomical references. Based on the intra-operative observations T1 mapping values were initially divided into two similar groups as normal or damaged.

For translating the $\mathrm{T} 1$ values into morphological findings we classified $\mathrm{T} 1$ values for peripheral and central zones: normal and below-normal based on the actual value of the T1. We used our own normative data from previously published studies of asymptomatic volunteers [29-31]. Accordingly, based on the mean value of $592.3 \mathrm{~ms}$ with a SD of $95.1 \mathrm{~ms}$ in the previous asymptomatic volunteer group, T1 values $>500 \mathrm{~ms}$ were assumed to be normal and $\mathrm{T} 1$ values $\leq 500 \mathrm{~ms}$ were regarded as below-normal. Lower values indicated GAG loss due to cartilage degeneration.

Intra-operative analysis

Following safe surgical hip dislocation [32, 33], an approach that allows for examination of the entire joint during surgery, the femoral and acetabular cartilage was evaluated for any signs of damaged cartilage. These findings were documented and classified according to the macroscopic damage and the accurate zonal location clockwise on the same basis as in standard MRI and T1, as normal or damaged. All surgeries and intra-operative evaluations were performed by two senior orthopedic surgeons well trained in these procedures. A standard evaluation sheet (Fig. 1) was used during every case to document the exact size (peripheral and/or central) and nature of damage. Descriptions included surface injury, malacia, debonding, and cleavage [34]. Other findings, such as labrum pathologies, were not considered in this present study. In addition to the classification into normal and damaged, a sub-analysis of the damaged cartilage was performed for malacia versus debonding/cleavage as typical findings in FAI [34]. 
Fig. 1 Intra-operative reporting sheet documenting the exact site and nature of damage including surface injury, cartilage malacia, debonding, and cleavage

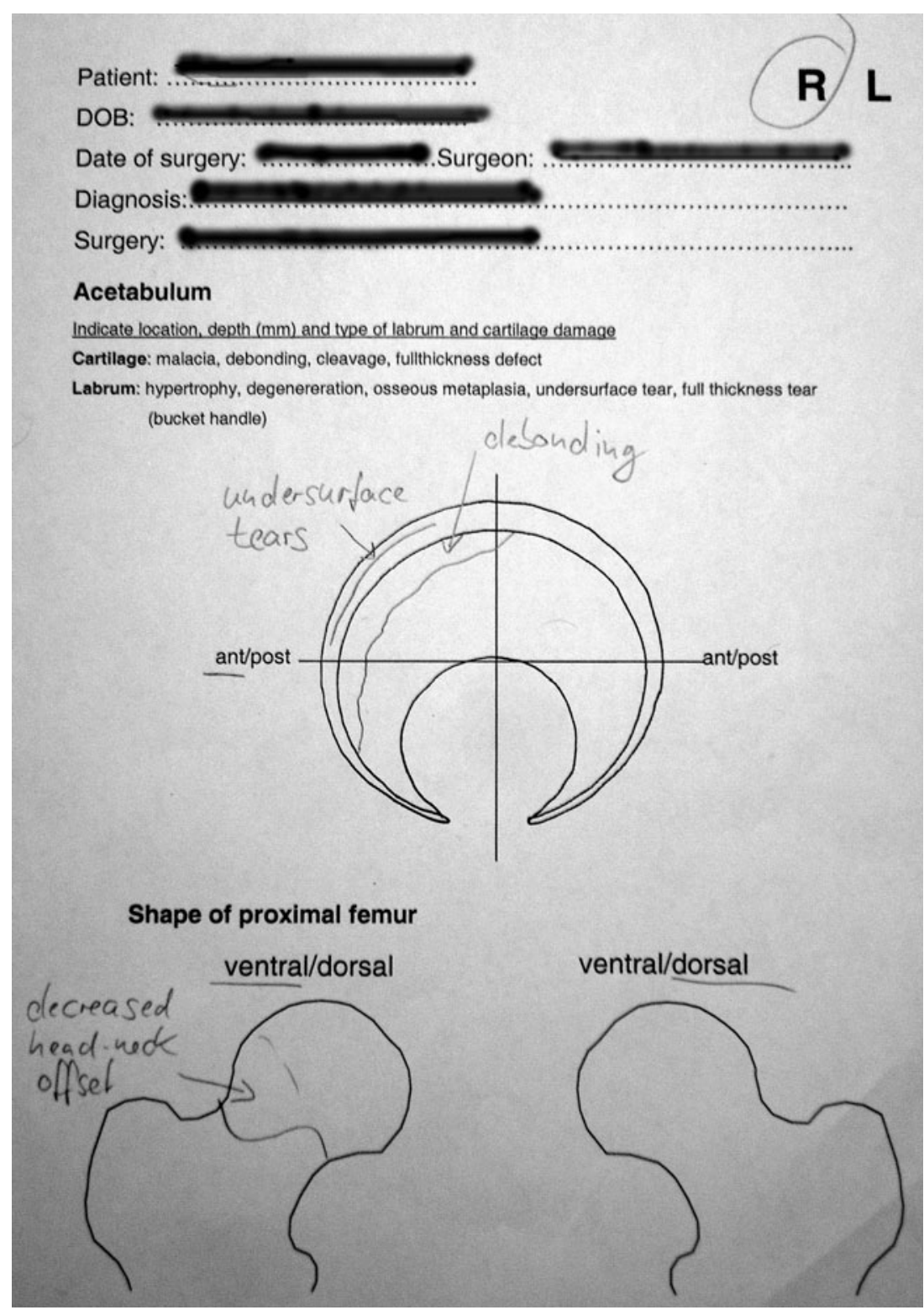

Statistical analysis

Two hundred and twenty-four zones (112 peripheral and 112 central) were evaluated with advanced imaging including standard MRI, T1-mapping and open intraoperative visualization.

Cartilage lesion frequencies noted with standard MRI and intra-operatively in the respective regions are shown as descriptive data. Corresponding $\mathrm{T} 1_{\mathrm{Gd}}$ values are expressed as mean values and SD. To assess the statistical differences between the $\mathrm{T} 1_{\mathrm{Gd}}$ values in cartilage categorized as normal by standard MRI or by intra-operative inspection and the $\mathrm{T} 1_{\mathrm{Gd}}$ values in cartilage categorized as damaged, Student's $t$ test (parametric variables, independent samples) [35] was utilized. The comparison between standard MRI findings and dGEMRIC with intra-operative findings was addressed by the Spearman's rank correlation coefficient (nonparametric measure of correlation) [36]. A $p$ value below 0.05 was considered to be statistically significant. Analysis was performed for each radial section, and for the peripheral and central zones in addition. SPSS software (Version 16.0; SPSS Institute, Chicago, IL, USA) was utilized for all statistical analyses.

\section{Results}

T1 dGEMRIC and intra-operative findings

One hundred and sixteen zones were rated as normal based on intra-operative findings. The mean $\mathrm{T} 1$ value of the respective zones that correlated to these was $510.1 \mathrm{~ms} \pm$ 
$141.2 \mathrm{~ms}$. Eighty zones were rated as having evidence of cartilage damage on intra-operative inspection. In this group the mean $\mathrm{T} 1$ value was $453.1 \mathrm{~ms} \pm 113.6 \mathrm{~ms}$. There was a statistically significant difference between the T1 values in the cartilage rated normal and those areas that had evidence of damage on intra-operative inspection $(p=0.003)$.

The correlation between dGEMRIC and intra-operative findings was very weak (Kappa correlation was $0.114, p<$ 0.126) [37]. Based on intra-operative visualization $56.4 \%$ zones were graded as normal and $43.6 \%$ were graded as damaged (Fig. 2). On the other hand, based on $\mathrm{T} 1$ assessment $31.4 \%$ of zones were graded as normal and $68.6 \%$ were graded as damaged. Details of the results are presented in Table 1.

Forty-one of the damaged cartilage zones were classified as malacia, and 39 as delamination (debonding/cleavage). Mean dGEMRIC index of the zones classified as malacia were significant lower (452.72 $\mathrm{ms} \pm 108.2)$ than zones classified as delamination of the cartilage $(551.3 \mathrm{~ms} \pm$ $107.9 ; p=0.02$ )

\section{Morphological MRI and intra-operative findings}

The correlation between standard MRI observations and intra-operative findings was moderate (Kappa correlation was $0.535, p<0.01$ ) [37]. Based on standard MRI, $68.6 \%$ zones were graded as normal, while $31.4 \%$ were graded as having evidence of damage (Figs. 3, 4). On the other hand, based on intra-operative visualization $56.4 \%$, zones were graded as normal and $43.6 \%$ were graded as having evidence of damage (Figs. 5, 6). Details of results are presented in Table 2.
Peripheral and central findings

Mean dGEMRIC T1 values were non-significantly ( $p=$ $0.469)$ lower in the peripheral area $(483.7 \pm 138.3 \mathrm{~ms})$ compared with those of the central area (510.4 $\pm 144.6 \mathrm{~ms})$.

Specificity for assessment of cartilage intra-operatively assessed as damaged with dGEMRIC was higher in the central zone (76.2\%) compared with the peripheral zone (60\%). Sensitivity was $37.3 \%$ in the central and $49.5 \%$ in the peripheral zone.

The correlation between standard MRI observations and dGEMRIC was higher for the central zone $(r=0.250, p=$ $0.008)$ compared with the peripheral zone $(r=0.093, p=$ 0.330 ). In the central zone based on standard MRI, $90 \%$ of zones were graded as normal, while $10 \%$ were graded as having evidence of damage. On the other hand, based on dGEMRIC 51.4\% zones were graded as normal and 48.6\% were graded as having evidence of damage.

\section{Discussion}

Current advances in imaging of cartilage are focused more and more on identifying better methods of delineating the biochemical changes of early cartilage damage. dGEMRIC and T1rho are currently the techniques that hold some promise in this direction. The key role of these techniques is their potential to detect early cartilage damage, which in turn is very helpful to clinicians for decision-making regarding timely intervention. The eventual goal of all pre-surgical imaging is better understanding of the disease process, enhanced communication between the radiologist
Fig. 2 a Morphological VIBE image and $\mathbf{b}$ corresponding $\mathrm{T} 1$ map depicting the regions of interest analysis for $\mathrm{T} 1$ measurements in the peripheral and central zones. Note that the $\mathrm{T} 1$ values are decreased in the peripheral zone $(382.6 \mathrm{~ms})$ compared with the central (500 ms)
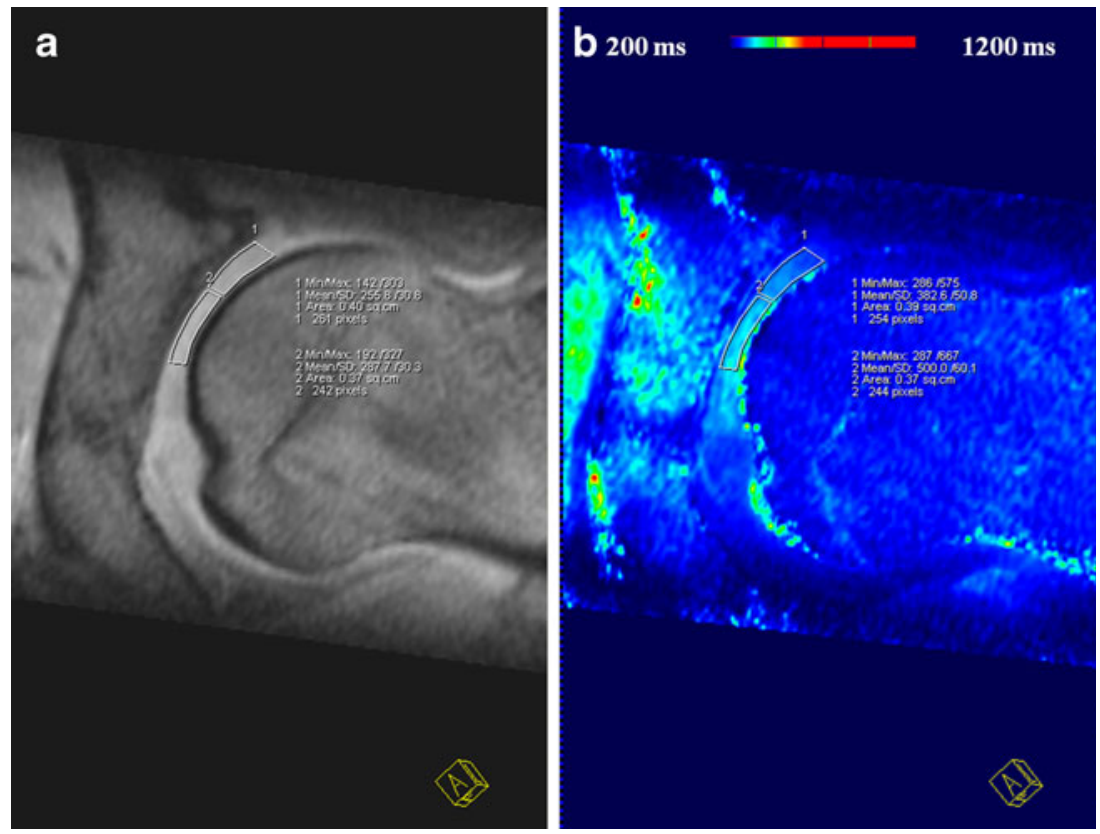
Table 1 The correlation between dGEMRIC and intra-operative findings was very weak (Kappa correlation was $0.114, p<0.126$ ). T1-mapping findings were more sensitive than intra-operative findings in delineating the status of the damaged cartilage. Based on intra- operative visualization, $56.4 \%$ of zones were graded as normal and $43.6 \%$ were graded as damaged. Based on $\mathrm{T} 1$ assessment, $31.4 \%$ of zones were graded as normal and $68.6 \%$ were graded as damaged

\begin{tabular}{|c|c|c|c|c|c|}
\hline & & & \multicolumn{3}{|c|}{ Cartilage grading by intra-operative inspection } \\
\hline & & & Normal & Damaged & Total \\
\hline \multirow[t]{6}{*}{ Cartilage grading by $\mathrm{T} 1$ analysis } & \multirow[t]{2}{*}{ Normal } & Number & 29 & 15 & 44 \\
\hline & & Percentage & 20.7 & 10.7 & 31.4 \\
\hline & \multirow[t]{2}{*}{ Damaged } & Number & 50 & 46 & 96 \\
\hline & & Percentage & 35.7 & 32.9 & 68.6 \\
\hline & \multirow[t]{2}{*}{ Total } & Number & 79 & 61 & 140 \\
\hline & & Percentage & 56.4 & 43.6 & 100 \\
\hline
\end{tabular}

and the treating clinician, and timely intervention (i.e., surgery when needed) that would help to positively change the outcome of the disease process (in this case FAI).

The purpose of this present study was to prospectively study the standard MRI and dGEMRIC findings in patients with symptomatic FAI undergoing surgical intervention and compare them with intra-operative findings to see if they were corroborative. To the best of our knowledge this is the first study that compares the pre-operative imaging findings of MRI using dGEMRIC for symptomatic FAI patients, with the subsequent intra-operative findings in the same cohort following safe surgical dislocation. It is important to realize that the focus was to identify the obvious morphological damage during intra-operative visualization; therefore, corroboration of the imaging findings included the extent of topographic damage rather than the degree of biochemical changes.

In our study we found that the correlation between standard MRI observations and intra-operative findings was

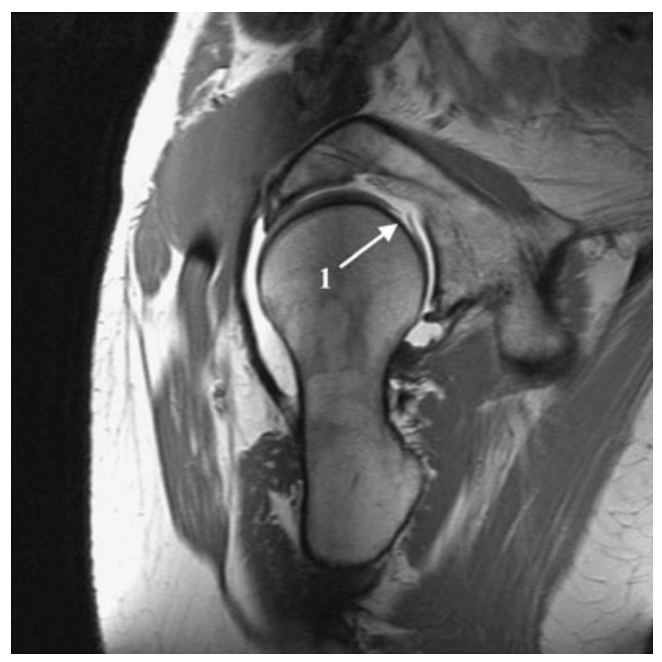

Fig. 3 Radial 2D TSE PD-weighted image depicting the central femoral head cartilage lesion with cartilage flap formation (arrow 1) in the posterior part moderate in that MRI underestimates intra-operative damage of the cartilage. Direct surgical (morphological) visualization has also been reported to be better than standard MR imaging in some previous reports [38, 39]. This in some ways may be related to the contrast, resolution, and technique of standard MR imaging, which is likely to miss some areas of cartilage (based on the thickness of the cut and the number of cuts obtained, as well as the averaging involved). Intra-operative visualization, on the other hand, allows more complete visualization of both the femoral and the acetabular sides of the joint and the macroscopic areas of suspected cartilage damage or areas with direct evidence of damage can be clearly identified.

Knowing what we know regarding the sensitivity of dGEMRIC to pick up early changes of cartilage damage (based on GAG content), it is not surprising that the correlation between dGEMRIC and intra-operative findings was moderate in our studies for a localized comparison of

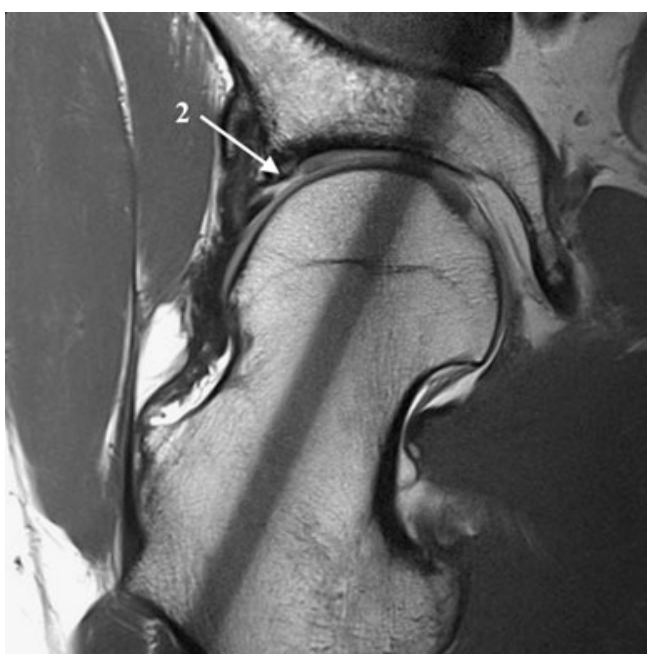

Fig. 4 Radial 2D TSE PD-weighted image depicting the cartilage damage at the acetabular rim in a cam-type patient in the superioranterior region (arrow 2) 


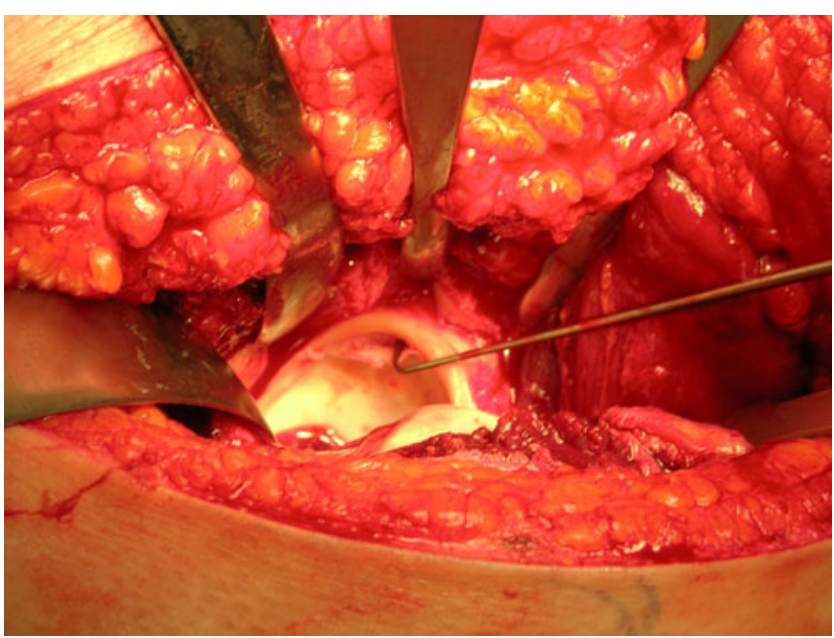

Fig. 5 Intra-operative photograph of the exposed acetabulum following safe surgical dislocation demonstrating the damage to acetabular cartilage and the rim tear consequent to the cam lesion. Note that the location is very classical for a cam lesion (anterior-superior)

different radial zones through the joint. Although there are no reports on specific timeframes of how and when the biochemical changes of cartilage damage become macroscopically visible in a hip joint with symptomatic FAI, presumably there is a time lag. This in turn is the likely reason why the surgeon's visualization of the extent of cartilage damage on intra-operative visualization notably underestimated the ability of dGEMRIC to do the same through the joint at cartilage surrounding the defect.

However, we noted significantly lower T1 dGEMRIC values in affected cartilage compared with normal cartilage in total. This is similar to studies by Bittersohl et al. [31], where decreased $\mathrm{T} 1$ values were also found in morphologically affected cartilage in patients with FAI using dGEMRIC. Within this study, the morphological changes were graded by MR arthrography, which are less sensitive based on our findings. In this context, it is of interest that areas of cartilage delamination showed a wide range of dGEMRIC index with significantly higher values compared with areas

Table 2 The correlation between standard MRI observations and intra-operative findings was moderate (Kappa correlation was 0.535 , $p<0.001)$. Intra-operative findings were noted to be more sensitive than standard MRI in delineating the morphological status of damaged

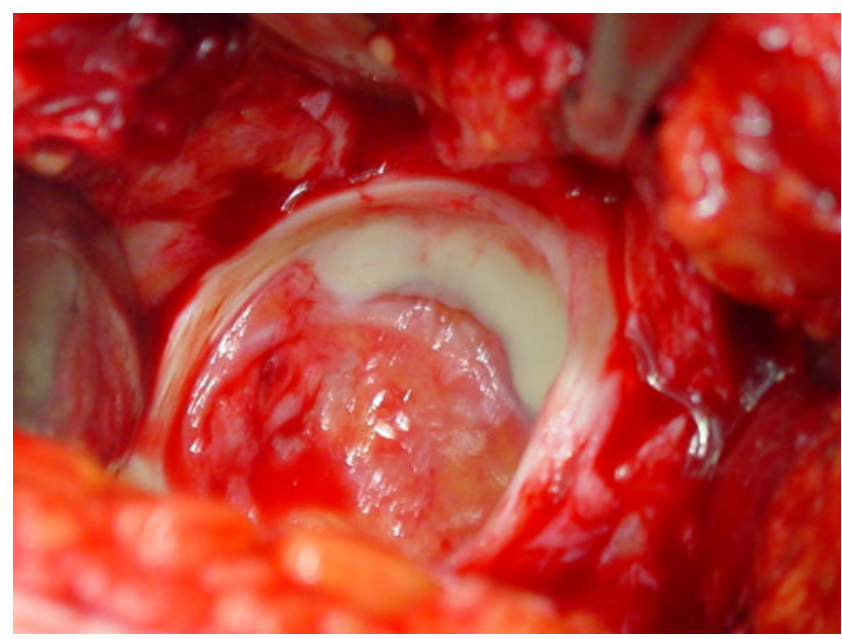

Fig. 6 Intra-operative photograph of the exposed acetabulum following safe surgical dislocation demonstrating full thickness cartilage abrasions

with cartilage malacia. As cartilage delamination is frequent in impingement [6, 37], this could be an additional explanation for the moderate correlation of dGEMRIC index and intra-operative findings. These differences in the dGEMRIC index for delaminated cartilage and the role of the outcome of surgery have to be further explored.

In addition, we assessed zonal differences in the dGEMRIC index from the peripheral to the central area. In the peripheral area, where typically cartilage lesions appear to be more frequent [6,37], more patients with decreased dGEMRIC index were also found. However, the decrease in dGEMRIC values was less localized compared with the intra-operative assessment of cartilage changes, which were located in the anterior to superior region. Therefore, the correlation with the intra-operative findings was weak compared with the central area, with fewer changes, but more localized. Therefore, dGEMRIC can add sensitivity in the detection of cartilage lesions; however, based on our data it also reflects whole joint being affected

cartilage. Based on standard MRI, $68.6 \%$ of zones were graded as normal, while $31.4 \%$ were graded as showing evidence of damage. Based on intra-operative visualization, $56.4 \%$ of zones were graded as normal and $43.6 \%$ were graded as having evidence of damage

\begin{tabular}{|c|c|c|c|c|c|}
\hline & & & \multicolumn{3}{|c|}{ Cartilage grading by intra-operative inspection } \\
\hline & & & Normal & Damaged & Total \\
\hline \multirow[t]{6}{*}{ Cartilage grading by standard MRI } & \multirow[t]{2}{*}{ Normal } & Number & 72 & 24 & 96 \\
\hline & & Percentage & 51.4 & 17.1 & 68.6 \\
\hline & \multirow[t]{2}{*}{ Damaged } & Number & 7 & 37 & 44 \\
\hline & & Percentage & 5.0 & 26.4 & 31.4 \\
\hline & \multirow[t]{2}{*}{ Total } & Number & 79 & 61 & 140 \\
\hline & & Percentage & 56.4 & 43.6 & 100 \\
\hline
\end{tabular}


because of impingement disease. Further studies have to show how much this changes the impact of the onset or preservation of further degeneration, similar to dGEMRIC data available for hip dysplasia [17].

There are limitations to this study. Our study population was relatively small. Although this was a limitation the prospective nature of the study offers strength to the data; therefore, these findings are preliminary and have to be confirmed in larger trials. One major limitation was the absence of an additional diagnostic gold standard in the form of histological analysis or intra-operative stiffness measurements, which better attempt to classify cartilage structure. In addition, we made no attempt to differentiate between acetabular and femoral cartilage as it was not possible to separate the two cartilage layers at an image resolution obtained with $1.5 \mathrm{~T}$. Therefore, in our study ROI analysis included acetabular and femoral cartilage as one combined entity. In some areas of severe or considerable damage it was difficult to differentiate between joint effusion and cartilage. ROI analysis obtains only mean values that represent the entire encircled area and therefore minor, but remarkable changes may have been possibly underestimated. However, this is the current limitation of the clinical use of dGEMRIC at $1.5 \mathrm{~T}$ and therefore the current status for comparison with intra-operative findings. Finally, there may have been some minor subjective variations in reading the intra-operative damage/changes by the surgeons as a part of human error.

In summary, dGEMRIC as a modality of advanced imaging of cartilage damage has the potential to significantly enhance capabilities in identifying and delineating topographically the areas of cartilage degeneration in hip joints of symptomatic patients with FAI. However, these changes are different to macroscopic changes found within surgery and dGEMRIC does not increase the diagnostic sensitivity of macroscopic changes. Therefore, the value and the role in surgical treatment planning has to be further defined based on outcome.

Conflict of interest The authors declare that there is no conflict of interest.

\section{References}

1. Ganz R, Parvizi J, Beck M, Leunig M, Notzli H, Siebenrock KA. Femoroacetabular impingement: a cause for osteoarthritis of the hip. Clin Orthop Relat Res. 2003;(417):112-20.

2. Murphy S, Tannast M, Kim YJ, Buly R, Millis MB. Debridement of the adult hip for femoroacetabular impingement: indications and preliminary clinical results. Clin Orthop Relat Res. 2004; (429): 178-81.
3. Wagner S, Hofstetter W, Chiquet M, Mainil-Varlet P, Stauffer E, Ganz R, et al. Early osteoarthritic changes of human femoral head cartilage subsequent to femoro-acetabular impingement. Osteoarthritis Cartilage. 2003;11:508-18.

4. Beck M, Leunig M, Parvizi J, Boutier V, Wyss D, Ganz R. Anterior femoroacetabular impingement. II. Midterm results of surgical treatment. Clin Orthop Relat Res. 2004;(418):67-73.

5. Spencer S, Millis MB, Kim YJ. Early results of treatment of hip impingement syndrome in slipped capital femoral epiphysis and pistol grip deformity of the femoral head-neck junction using the surgical dislocation technique. J Pediatr Orthop. 2006;26:281-5.

6. Pfirrmann CW, Mengiardi B, Dora C, Kalberer F, Zanetti M, Hodler J. Cam and pincer femoroacetabular impingement: characteristic MR arthrographic findings in 50 patients. Radiology. 2006;240:778-85.

7. Czerny C, Hofmann S, Neuhold A, Tschauner C, Engel A, Recht $\mathrm{MP}$, et al. Lesions of the acetabular labrum: accuracy of MR imaging and MR arthrography in detection and staging. Radiology. 1996;200:225-30.

8. Locher S, Werlen S, Leunig M, Ganz R. MR-Arthrography with radial sequences for visualization of early hip pathology not visible on plain radiographs. Z Orthop Ihre Grenzgeb. 2002;140:52-7.

9. Petersilge CA. MR arthrography for evaluation of the acetabular labrum. Skeletal Radiol. 2001;30:423-30.

10. Petersilge CA, Haque MA, Petersilge WJ, Lewin JS, Lieberman JM, Buly R. Acetabular labral tears: evaluation with MR arthrography. Radiology. 1996;200:231-5.

11. Bashir A, Gray ML, Hartke J, Burstein D. Nondestructive imaging of human cartilage glycosaminoglycan concentration by MRI. Magn Reson Med. 1999;41:857-65.

12. Nieminen MT, Rieppo J, Silvennoinen J, Toyras J, Hakumaki JM, Hyttinen MM, et al. Spatial assessment of articular cartilage proteoglycans with Gd-DTPA-enhanced T1 imaging. Magn Reson Med. 2002;48:640-8.

13. Bashir A, Gray ML, Boutin RD, Burstein D. Glycosaminoglycan in articular cartilage: in vivo assessment with delayed Gd(DTPA) (2-)-enhanced MR imaging. Radiology. 1997;205:551-8.

14. Burstein D, Velyvis J, Scott KT, Stock KW, Kim YJ, Jaramillo D, et al. Protocol issues for delayed Gd(DTPA)(2-)-enhanced MRI (dGEMRIC) for clinical evaluation of articular cartilage. Magn Reson Med. 2001;45:36-41.

15. Cunningham T, Jessel R, Zurakowski D, Millis MB, Kim YJ. Delayed gadolinium-enhanced magnetic resonance imaging of cartilage to predict early failure of Bernese periacetabular osteotomy for hip dysplasia. J Bone Joint Surg Am. 2006;88:1540-8.

16. Gillis A, Bashir A, McKeon B, Scheller A, Gray ML, Burstein D. Magnetic resonance imaging of relative glycosaminoglycan distribution in patients with autologous chondrocyte transplants. Invest Radiol. 2001;36:743-8.

17. Kim YJ, Jaramillo D, Millis MB, Gray ML, Burstein D. Assessment of early osteoarthritis in hip dysplasia with delayed gadolinium-enhanced magnetic resonance imaging of cartilage. J Bone Joint Surg Am. 2003;85-A:1987-92.

18. Kurkijarvi JE, Mattila L, Ojala RO, Vasara AI, Jurvelin JS, Kiviranta I, et al. Evaluation of cartilage repair in the distal femur after autologous chondrocyte transplantation using T2 relaxation time and dGEMRIC. Osteoarthritis Cartilage. 2007;15:372-8.

19. Tiderius CJ, Olsson LE, de Verdier H, Leander P, Ekberg O, Dahlberg L. Gd-DTPA2)-enhanced MRI of femoral knee cartilage: a dose-response study in healthy volunteers. Magn Reson Med. 2001;46:1067-71.

20. Tiderius CJ, Olsson LE, Leander P, Ekberg O, Dahlberg L. Delayed gadolinium-enhanced MRI of cartilage (dGEMRIC) in early knee osteoarthritis. Magn Reson Med. 2003;49:488-92. 
21. Tiderius CJ, Svensson J, Leander P, Ola T, Dahlberg L. dGEMRIC (delayed gadolinium-enhanced MRI of cartilage) indicates adaptive capacity of human knee cartilage. Magn Reson Med. 2004;51:286-90.

22. Trattnig S, Mamisch TC, Pinker K, Domayer S, Szomolanyi P, Marlovits S, et al. Differentiating normal hyaline cartilage from post-surgical repair tissue using fast gradient echo imaging in delayed gadolinium-enhanced MRI (dGEMRIC) at 3 Tesla. Eur Radiol. 2008;18:1251-9.

23. Vasara AI, Nieminen MT, Jurvelin JS, Peterson L, Lindahl A, Kiviranta I. Indentation stiffness of repair tissue after autologous chondrocyte transplantation. Clin Orthop Relat Res. 2005; (433):233-42.

24. Williams A, Sharma L, McKenzie CA, Prasad PV, Burstein D. Delayed gadolinium-enhanced magnetic resonance imaging of cartilage in knee osteoarthritis: findings at different radiographic stages of disease and relationship to malalignment. Arthritis Rheum. 2005;52:3528-35.

25. Cova M, Toffanin R. MR microscopy of hyaline cartilage: current status. Eur Radiol. 2002;12:814-23.

26. Poole AR, Kojima T, Yasuda T, Mwale F, Kobayashi M, Laverty S. Composition and structure of articular cartilage: a template for tissue repair. Clin Orthop Relat Res. 2001;(391 Suppl):S26-33.

27. Buckwalter JA, Roughley PJ, Rosenberg LC. Age-related changes in cartilage proteoglycans: quantitative electron microscopic studies. Microsc Res Tech. 1994;28:398-408.

28. Venn M, Maroudas A. Chemical composition and swelling of normal and osteoarthrotic femoral head cartilage. I. Chemical composition. Ann Rheum Dis. 1977;36:121-9.

29. Bittersohl B, Hosalkar HS, Haamberg T, Kim YJ, Werlen S, Siebenrock KA, et al. Reproducibility of dGEMRIC in assessment of hip joint cartilage: a prospective study. J Magn Reson Imaging. 2009;30:224-8.

30. Bittersohl B, Hosalkar HS, Hughes T, Kim YJ, Werlen S, Siebenrock KA, et al. Feasibility of $\mathrm{T}(2)\left(^{*}\right)$ mapping for the evaluation of hip joint cartilage at $1.5 \mathrm{~T}$ using a three-dimensional
(3D), gradient-echo (GRE) sequence: a prospective study. Magn Reson Med. 2009;62:896-901.

31. Bittersohl B, Steppacher S, Haamberg T, Kim YJ, Werlen S, Beck $\mathrm{M}$, et al. Cartilage damage in femoroacetabular impingement (FAI): preliminary results on comparison of standard diagnostic vs delayed gadolinium-enhanced magnetic resonance imaging of cartilage (dGEMRIC). Osteoarthritis Cartilage. 2009;17:1297306.

32. Ganz R, Gill TJ, Gautier E, Ganz K, Krugel N, Berlemann U. Surgical dislocation of the adult hip: a technique with full access to the femoral head and acetabulum without the risk of avascular necrosis. J Bone Joint Surg Br. 2001;83:1119-24.

33. Lavigne M, Parvizi J, Beck M, Siebenrock KA, Ganz R, Leunig M. Anterior femoroacetabular impingement. I. Techniques of joint preserving surgery. Clin Orthop Relat Res. 2004;(418):616.

34. Beck M, Kalhor M, Leunig M, Ganz R. Hip morphology influences the pattern of damage to the acetabular cartilage: femoroacetabular impingement as a cause of early osteoarthritis of the hip. J Bone Joint Surg Br. 2005;87:1012-8.

35. Bowalekar SK. Statistics in medical research. IV. Sampling distribution, statistical testing of hypothesis and student's t-test. J Postgrad Med. 1994;40:46-51.

36. Myers JL, Well A. Research design and statistical analysis. In: Mahwah, NJ: Erlbaum; 2003.

37. Landis JR, Koch GG. An application of hierarchical kappa-type statistics in the assessment of majority agreement among multiple observers. Biometrics. 1977;33:363-74.

38. Knuesel PR, Pfirmann CW, Noetzli HP, Dora C, Zanetti M, Hodler J, et al. MR arthrography of the hip: diagnostic performance of a dedicated water-excitation 3D double-echo steady-state sequence to detect cartilage lesions. AJR Am J Roentgenol. 2004;183:1729-35.

39. Schmid MR, Notzli HP, Zanetti M, Wyss TF, Hodler J. Cartilage lesions in the hip: diagnostic effectiveness of MR arthrography. Radiology. 2003;226:382-6. 